right thigh with discoloration and bruising. The superficial epithelium had sloughed off. Her lungs, spleen, and liver were congested. Str pyogenes was isolated from her skin and subcutaneous tissue, but not from her heart blood or spleen. Her right hip showed no underlying abnormality.

This is another example of a patient with Str pyogenes infection who did not have a classical presentation. This case also emphasises the fulminating nature of the infection, as she died despite having been on appropriate high doses of benzylpenicillin for 20 hours.

Penelope J Redding

Department of Bacteriology and Immunology, Glasgow G11 6NT

\section{Comparison of neonatal management} methods for very low birthweight babies

SIR,-Dr E S Steiner and others (8 November, p 1237) have offered a very succinct paper examining the outcome of very low birthweight infants who did not require "special or intensive" care. I am unable to agree with their conclusions and am saddened by their hostility towards intensive perinatal care.

Clearly none of their infants required intensive care intervention and those that did died. Thus a population of "well babies" remained, of whom only $52 \%$ were normal at school age. Surely these results are disastrous and must indicate that more intensive care facilities are required at this unit.

A more useful comparison would be a similar population at University College Hospital or Hammersmith Hospital-that is, very low birthweight infants who did not require intensive therapy after birth and who were managed by minimal handling only. The figures quoted from these other units included babies ventilated for hyaline membrane disease. I would be very interested to learn whether Dr Pamela Davies at Hammersmith Hospital has been able to differentiate non-ventilated very low birthweight infants in her follow-up studies.

G J REYNOLDS

Institute of Child Health, Alder Hey Children's Hospital,
Liverpool L12 2AP

* * We sent this letter to Dr Davies, who replies below.-ED, $B M \mathcal{~}$.

SIR,-Thank you for giving me the opportunity to reply to Dr Reynolds's query about the outcome for non-ventilated very low birthweight infants. In the 15-year (1961-75) survey of infants weighing $\leqslant 1500 \mathrm{~g}$ at their birth in Hammersmith Hospital ${ }^{1}$ to which Dr E S Steiner and his colleagues refer (8 November, p 1237) 148 of the total of 357 were ventilated, and $94 \%$ of them died. The fate of the 209 non-ventilated infants was as follows: $70(33.5 \%)$ died in the neonatal period, 5 $(2 \cdot 4 \%)$ later, $5(2 \cdot 4 \%)$ were untraced, 17 $(8.1 \%)$ had major and $20(9.6 \%)$ minor handicap as defined, ${ }^{1}$ and $92(44.0 \%)$ were apparently normal. The figures for major and minor handicap reported by Dr Steiner and his colleagues were $5.5 \%$ and $12.3 \%$ respectively.

\section{P A Davies}

\section{Hammersmith Hospital}

1 Jones RAK, Cummins M, Davies PA. Lancet 1979; i:1332-5.

\section{Intrinsic hazard of breech presentation}

SIR,-The management of breech delivery continues to be widely debated. Caesarean section is increasingly used to deliver mature breech babies. Some obstetricians advocate abdominal delivery for those between $\mathbf{3 2}$ and 37 weeks while others include very small prematures-that is, those between 26 and 32 weeks. Presumably the operation referred to has always been the lower-segment one.

Mr John P Calvert (15 November, p 1319) now suggests that ill, small breech babies should be delivered by classical section because the fetal head might be delivered more quickly for resuscitation. Surely the risks of such a policy must far outweigh the advantages. The uterine wound is often larger with the classical procedure and when the placenta is anterior the blood loss at operation will often demand transfusion Paralytic ileus, generalised peritonitis, and occasionally acute small bowel obstruction are complications to be reckoned with in the puerperium. ${ }^{1}$ But the over-riding argument against the use of classical section for small compromised breech is that the scar can and does rupture in any subsequent pregnancy with the risk of maternal death and a fetal mortality rate of approximately $75 \%{ }^{2}$ The results quoted by $\mathrm{Dr}$ Calvert suggest that if a breech baby has to be delivered by section then a colleague skilled in resuscitation must be in the theatre. Perhaps the operation should be performed under epidural block using all methods to prevent the supine hypotensive syndrome. Certainly all obstetric anaesthetic skills are essential. The abdomen should be opened by a mid-line incision an the fetus delivered through an adequate transverse incision in the lower segment.

Perinatal statistics have become too important. It is wiser to deliver a young teenager of a 35-week breech vaginally using an epidural block than to seriously prejudice her obstetric career let alone her life by performing a classical section. Similarly, if the cord prolapses during the labour of a smal premature breech baby it is often sensible not to operate. A stillborn 30 -week fetus with the uterus left intact is preferable in a young woman than to leave her with a scarred upper segment and a brain-damaged baby.

New Cross Hospital

Alan M SMITH

Wolverhampton

${ }^{1}$ Myerscough, P. Munro Kerr's operative obstetrics. 8th edn. London: Balliere Tindall, 1977:511. Dewhurst CJ. Fournal of Obstetrics and Gynaecology of the British Empire 1957;64:113-8.

\section{Kidney biopsy}

SIR,-The juxtaposition of two letters (4 October, p 945) containing similar statistics, but supporting two different techniques for kidney localisation prior to biopsy, will not help those trying to decide which method they should use. Our letter from Nottingham was not written with the knowledge that it would be adjacent to the vigorous advocacy for urographic localisation put forward in the Bristol letter. It is clear that both techniques are satisfactory when used by experienced staff, and a choice between the two cannot be made on the basis that one is more likely to be successful, or is less prone to complications.

The merits of ultrasound are in part logistic; it is quicker to establish the position of the kidney than with urography. The technique is especially useful iwhen it is uncertain if urography will demonstrate the kidney. It provides the operator with the distance of the kidney capsule from the skin. In our opinion the role of biopsy with urography will be for the few failures under ultrasound, when the depth of the kidney will already be known.

It seems certain that very occasional severe renal haemorrhage will occur with all methods. It is surprising that neither the original article nor the correspondence comments on the treatment of this complication by selective embolisation of the bleeding kidney. We hesitated to be advocates of this technique with only two severe haemorrhages to report. The first followed a "blind" biopsy in acute renal failure before the era of selective embolisation and nephrectomy was performed after 10 days of bleeding. In the second patient, with chronic renal failure and before the use of ultrasound, a selective embolisation stopped prolonged and severe bleeding with no detectable impairment of renal function. It is important that those performing renal biopsy should be aware of the technique, as it appears much preferable to nephrectomy. Its availability has reduced to some extent the caution with which we recommend renal biopsy.

MARTIN S KNAPP

R P BURDEN

D H ROSE

Peter Davies

City Hospital

Nottingham NG5 1PD

\section{Renal aneurysms in rejected renal} transplants

SIR,-The finding of multiple small intrarenal aneurysms by Dr J F. Walker and others (8 November, p 1251) prompted us to review the renal arteriograms performed in our unit during the past seven years with a view to determining the incidence of such aneurysms, both in general and in our own transplant series.

In this period 918 renal arterial studies were performed with selective renal arteriography, flush aortography, or a combination of both techniques, depending on the clinical indication for the study. In some examinations (for example, transplanted kidneys) just one kidney was examined but in most others both kidneys. Indications for the procedure included suspected tumour (262 kidneys), suspected rejection or arterial stenosis in a renal transplant (136 kidneys), suspected polyarteritis nodosa (102 kidneys), the assessment of prospective renal donors (162 kidneys), and miscellaneous pathology (hypertension, abnormal urogram, haematuria, etc, 256 kidneys). In the total series of 918 organs studied, multiple small intraparenchymal aneurysms were demonstrated in 24 kidneys (14 patients); four of these kidneys (four patients) were rejected transplants and the other 20 (10 patients) were in cases of polyarteritis nodosa Aneurysms were also seen in association with hypertension, stenosis, and atherosclerosis and following renal biopsy, renal surgery, or renal trauma. These aneurysms all affected the large intrarenal arteries or the extrarenal arteries, and were usually solitary; their appearance was quite distinct from the multiple small lesions of polyarteritis or transplant rejection. Bizarre aneurysmal vessels were also seen in the pathological circulation of renal tumours; the lesions in these cases were confined to the region of the tumour.

It has been noted that aneurysms were demonstrated in four out of 136 transplanted kidneys examined arteriographically ( $3 \%$ ). A furthe 